\title{
Microstructural Analysis of CdTe Thin Films using EBSD and FIB
}

\author{
M.M. Nowell,* S.I. Wright, ${ }^{*}$ and J.O. Carpenter* \\ * EDAX-TSL, 392 E 12300 S Suite H, Draper, UT 84020 USA
}

The driving force to develop technologies and materials for alternative energy production has been increasing due to economic and political factors. The development and application of photovoltaic (PV) solar cell materials for converting light into electricity is well documented, and has demonstrated a significant growth rate that is expected to continue. Due to the cost of silicon wafers used in traditional solar cells, thin film based alternatives using less material and alternative substrates have been developed. Cadmium telluride (CdTe) is one such alternative, and has been used to produce commercial solar cells. However research is currently continuing to improve the efficiency of these devices.

One area of research is determining the role of grain boundaries in device performance. Typically grain boundaries are considered defects within a material. However polycrystalline CdTe cells have exhibited higher efficiencies than single crystal cells and the reason for this is not well understood. Grain boundaries can act as diffusion pathways for different elements, and elemental segregation can occur. This can result in a change in electrical properties of the boundaries.

Electron backscatter diffraction (EBSD) and Orientation Imaging Microscopy (OIM) is a well suited analytical microanalysis technique for investigating this type of thin film material. By providing spatially specific orientation data on a sub-micron scale, it is possible to measure the grain boundary structure with enough data for statistical significance. This allows for the identification and differentiation of different grain boundary types such as twin boundaries, low angle boundaries, and random high angle boundaries. These different boundary types may have significantly different electrical properties and their distributions could be varied through grain boundary engineering principles.

Often CdTe films have a surface roughness that limits EBSD collection efficiency. Previous work has shown that this efficiency can be improved through mechanical and broad beam ion polishing [1]. An alternative approach is to use a Focused Ion Beam (FIB) for site specific preparation. Figure 1 shows EBSD Image Quality (IQ) maps from ion broad beam and FIB prepared surfaces, where better grain contrast is obtained using the FIB. By varying the glancing angle of the FIB, it is also possible to profile the microstructure as a function of film thickness. FIB has also been used to examine grain boundary electrical behavior at specific sites using Electron Beam Induced Current (EBIC) measurements [2].

In addition to grain boundary structure, EBSD provides information on orientation, grain size and shape, and phase distributions. Figure 2 shows a Local Orientation Spread Map with a bimodal grain size structure of small strained grains and larger strain free twinned grains from a partially recrystallized CdTe film. This data can help understand the evolution that occurs during thermal processing.

References 
[1] H.R. Moutinho et al., J. Vac. Sci. Technol. A 26(4) (2008) 1068.

[2] M. Terheggen et al., Interface Science 12 (2004) 259.

[3] J. Poortmans and V. Arkhipov, Thin Film Solar Cells: Fabrication, Characterization, and Applications, John Wiley \& Sons, Ltd., West Sussex, England 2006.

[4] T.L. Matteson et al., Journal of Electronic Materials 31 (2002) 33.

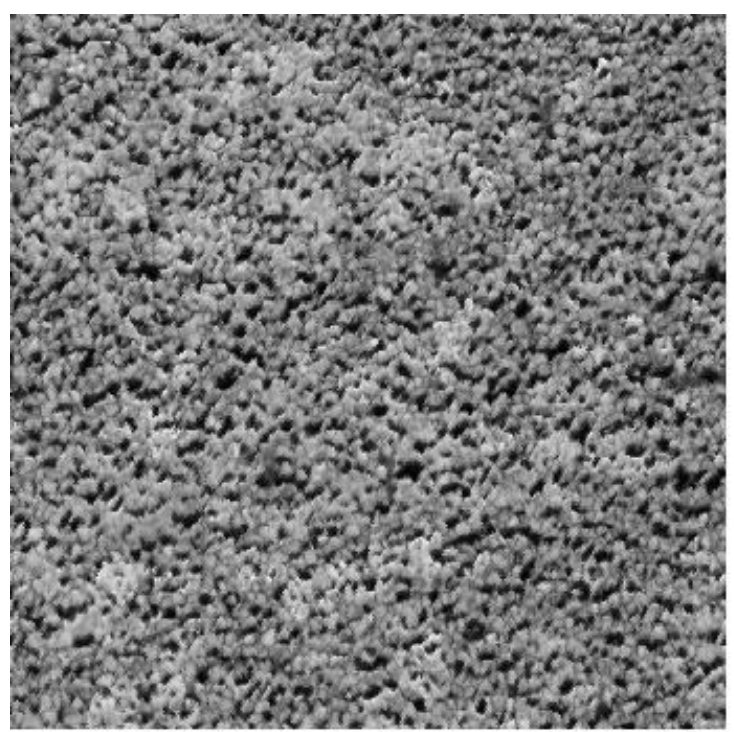

$10 \mu \mathrm{m}$

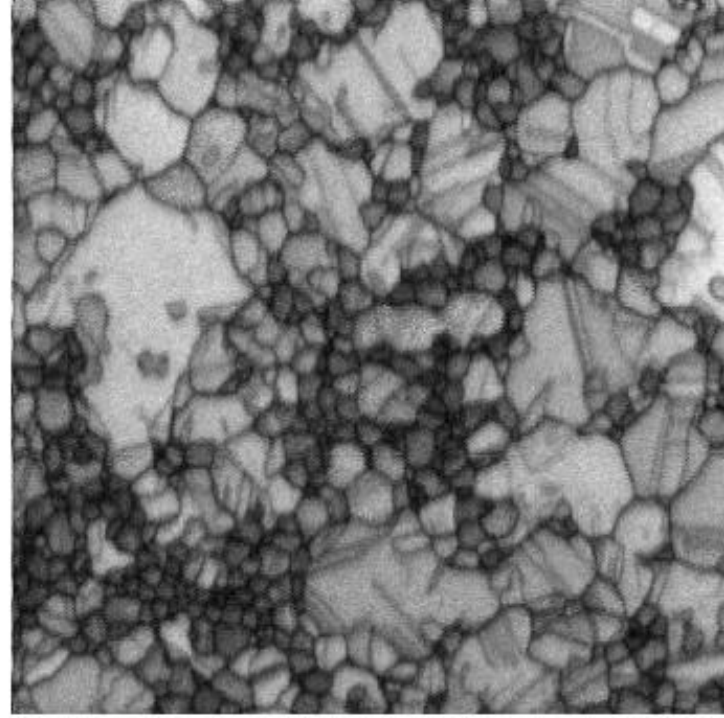

$10 \mu \mathrm{m}$

FIG. 1. EBSD Image Quality maps of a Broad Ion Beam prepared surface (left) and a Focused Ion Beam (FIB) prepared surface (right). Note the FIB surface provides better orientation and grain boundary contrast and less topography.

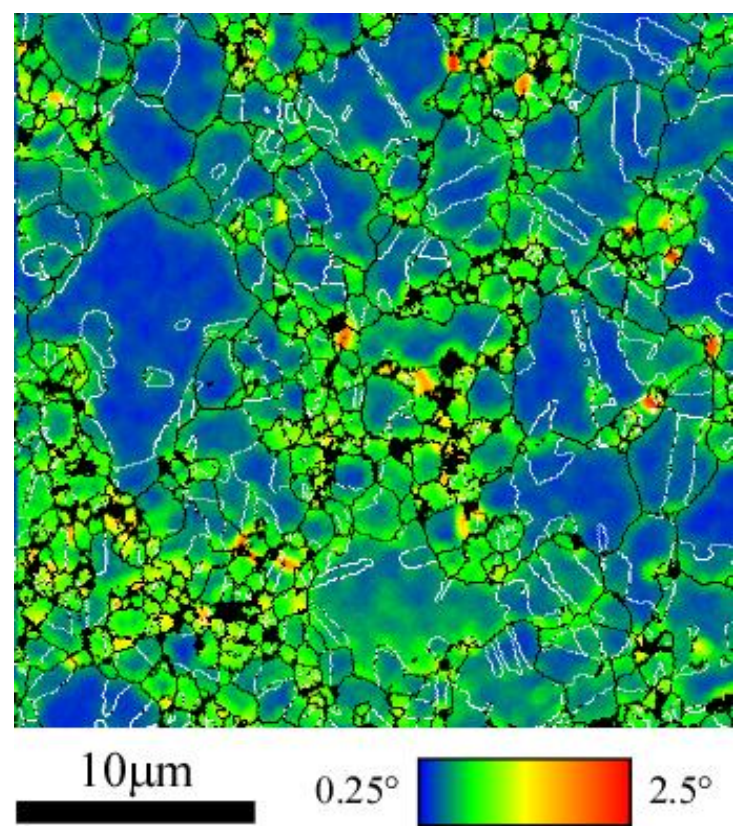

FIG. 2. Local Orientation Spread map ( $4^{\text {th }}$ nearest neighbor kernel) with twin boundaries shown in white and random high angle boundaries shown in black. 